\title{
After Meals
}

National Cancer Institute

\section{Source}

National Cancer Institute. After Meals. NCI Thesaurus. Code C64594.

After the ingestion of food. 